\title{
IMPORTÂNCIA DO PROCESSO DE COMUNICAÇÃO NA ASSISTENCIA DE ENFERMAGEM
}

\author{
Maguida Costa Stefanelli*
}

STEFANELLI, M. C. Importância do processo de comunicação na assistência de enfermagem. Rev. Esc. Enf. USP, São Paulo, 15(3):239-245, 1981.

A autora apresenta uma revisão de literatura sobre a importância da comunicação na interação enfermeira-paciente, qualquer que seja sua área de atuação. Focaliza sumariamente opiniões sobre conceito de comunicação, comunicação terapêutica e necessidade de pesquisas de enfermagem neste campo.

\section{INTRODUÇĀO}

Observando estudantes de enfermagem no desempenho de suas funções, constatamos que uma das dificuldades que a maioria deles apresenta é estabelecer diálogo significativo com o paciente. Quando o paciente começa a falar de suas ááreas de real preocupação, a aluna torna-se ansiosa e encerra a conversação. Comenta, depois, que a encerrou porque não sabia o que falar ou como dar seqüência ao assunto.

Na disciplina Enfermagem Psiquiátrica, é dada ênfase ao aspecto da comunicação enfermeira-paciente. São ministradas aulas sobre conceitos básicos de comunicação e técnicas terapêuticas de comunicação. As alunas, inicialmente, resistem ao uso das técnicas alegando que estas tornam o relacionamento com o paciente muito formal. Ao término do estudo da disciplina, porém, o aluno, embora ainda não consiga usar adequadamente todas as técnicas terapêuticas apresentadas, comenta que seria muito mais intereressante ter aprendido estas no início do curso de enfermagem, pois acha que com isto poderia ter evitado várias situaçōes desagradáveis e mesmo ajudado de modo mais eficiente o paciente. Reconhece, portanto, que o uso de técnicas terapêuticas de comunicação é útil e necessário em qualquer área de enfermagem e não só em Enfermagem Psiquiátrica.

0 presente artigo tem por objetivo ressaltar a importância da comunicação para a assistência de enfermagem e estimular as colegas, docentes ou não, e os alunos para que pensem mais ativamente sobre a importância da comunicação verbal e não verbal que se desenvolve entre eles e o paciente.

\section{CONCEITO DE COMUNICAÇÃO}

Comunicação é a função humana que torna possível a uma pessoa relacionar-se com outra (RUESCH, 1957). 0 significado da comunicação abrange todos

\footnotetext{
* Professor Assistente do Departamento de Enfermagem Materno-Infantll e Psiquisitrica da EEUSP. disciplina Enfermagem Psiquiátrica. Mestre em Enfermagem.
} 
os procedimentos por meio dos quais um ser humano pode afetar outro. Esses procedimentos incluem tanto a comunicação verbal como a não verbal. $O$ conceito abrange duas ou mais pessoas em interação e um intercâmbio de mensagens, em determinado contexto. Para que a comunicação seja efetiva, é necessário que o receptor compreenda a mensagem e dê uma resposta. A percepção da mensagem influi profundamente na pessoa e pode provocar mudança da conduta humana (RUESCH, 1964). Este afirma, ainda, que o fracasso na comunicação provoca frustração, a qual, se prolongada ou intensa, torna pensamentos, sentimentos e reações progressivamente inapropriados e desorganizados; isto reduz a capacidade do individuo para o relacionamento interpessoal.

Comunicação, segundo HOFLING et alii (1970), é um processo realizado em dois sentidos, de modo recíproco, que transmite mensagens entre indivíduos. A resposta à mensagem provoca, a curto ou longo prazo, mudança no modo de sentir, pensar ou atuar de uma pessoa.

\section{IMPORTÂNCIA DA COMUNICAÇÃO}

RUESCH (1964), o pioneiro da comunicação terapêutica, e SULLIVAN (1953), o introdutor da teoria interpessoal, na psiquiatria, colocam a doença mental como distúrbî̀ no processo de comunicação da pessoa e como perturbação no relacionamento interpessoal, respectivamente. Torna-se, assim, fácil para a enfermeira perceber a importância da comunicação na assistência de enfermagem, já que, dos membros da equipe dedicada à assistência do paciente, é ela que passa mais tempo junto ao mesmo e que mais oportunidades tem para com ele interagir.

Vários autores colocam em evidência a importância da comunicação na assistência de enfermagem; no cuidado do doente mental, o uso de técnicas terapêuticas de comunicação, na interação enfermeira-paciente, tornou-se o foco de atenção.

Florence Nightingale, em 1859, já demonstrava preocupação em relação à comunicação associada à assistência de enfermagem. Ela faz comentários sobre certos tipos de diálogos e perguntas vagas que provocam respostas imprecisas (NIGHTINGALE, 1946).

Segundo TUDOR (1970), é esperado, cada vez mais, que enfermeiras desenvolvam habilidades interpessoais de modo útil e terapêutico com pacientes em hospital psiquiátrico. A ação da enfermeira deve: 1. facilitar a comunicação do paciente com os que o circundam, visto ser a doença mental, em parte, um defeito de comunicação; 2 . facilitar a participação social, uma vez que a caraterística predominante do doente mental é o isolamento; 3. providenciar para que as necessidades do paciente sejam satisfeitas. A citada autora publicou este artigo pela primeira vez na revista Psychiatry, em 1952, porque não havia, na época, periódico de enfermagem que comportasse artigo tão extenso e especializado. Essa publicação provocou mudança decisiva na assistência de enfermagem psiquiátrica. A enfermeira passou a cuidar do paciente como uma pessoa inserida em seu meio.

De acordo com PEPLAU (1952), as operaçōes envolvidas num processo de enfermagem são tanto técnicas como interpessoais, mas o processo em si não pode 
ser definido como técnico. Enfermagem é um relacionamento humano, entre o indivíduo que está doente ou necessitando de ajuda de profissionais de saúde e a enfermeira deve ser preparada para responder a essas necessidades. A enfermagem torna-se um processo educativo e terapêtico quando há, entre enfermeira e paciente, respeito mútuo. Os fatores que norteiam o relacionamento enfermeira-paciente são inúmeros e complexos e não há fórmulas mágicas para enfrentá-los, uma vez que cada situação interpessoal é única e nunca poderá se repetir. Daí podemos inferir a necessidade da enfermeira fazer uso consciente da comunicação em toda interação com o paciente, para tentar compreender e identificar suas necessidades.

KEMPF (1957), entre as qualidades que considera importantes para a enfermeira, destaca a habilidade em comunicar-se com o outro. A enfermeira deve demonstrar sensibilidade à comunicação não falada e à capacidade de ouvir atentamente, saber o que falar e quando falar, em linguagem clara e acessível ao paciente e seus familiares.

A enfermeira tem que possuir habilidade interpessoal para ajudar o paciente quando este é assistido na unidade de internação. Como participante do processo de interação com o paciente, ela tem que estudar não só a comunicação do mesmo como a sua própria e aprender a usar a sua experiência interpessoal (GREGG, 1954). Ela tem que analisar o que é útil, ou não, nas suas interações com o paciente, para prover experiência interpessoal terapêutica (GREGG, 1963).

WIEDENBACH (1963) coloca a enfermagem como a arte de ajudar o paciente. Para a autora, se o objetivo da enfermeira é ajudar um determinado paciente, ela tem que centrar sua atenção na real necessidade de ajuda deste. Para atender esta necessidade ela lança mão de todo seu potencial e habilidades, especialmente as relacionadas à comunicação com pacientes.

De suma importância para a enfermagem é a habilidade em comunicar-se com pacientes, como afirma FUERST \& WOLFF (1964). Essas autoras consideram que, se este aspecto da enfermagem for negligenciado, estará sendo negada à enfermeira a oportunidade de conhecer aquele que está sob seu cuidado e de obter satisfação para ambos.

Ressalta MORRIN (1965) a necessidade da enfermeira tornar sua comunicação eficaz. Afirma que a base para o tratamento do paciente vem de dados colhidos da observação e da comunicação com pacientes. Reconhece ser o aprendizado da habilidade de comunicação um processo gradual e que requer atenção durante toda a vida profissional da enfermeira.

MEYERS (1964) usou, em uma pesquisa, três tipos de comunicação ao ministrar um cuidado de enfermagem. Na primeira vez, a enfermeira ministrou o cuidado sem dizer nada ao paciente; na segunda vez, conversou sobre assuntos superficiais, não pertinentes ao cuidado; a terceira vez, demonstrou interesse pelo paciente, explicou-lhe o que estava fazendo e o porquê. Concluiu que o último tipo de comunicação, que chamou de estruturado, foi o mais benéfico para o paciente; fez com que ele se sentisse tratado como pessoa e provocou diminuição da ansiedade, causada pelos problemas inerentes à hospitalização. 
Só pelo uso de comunicação efetiva poderemos atingir os objetivos da relação de ajuda enfermeira-paciente, citados por TRAVELBEE (1969). A enfermeira ajuda o paciente a enfrentar seus problemas atuais, conceituá-los, perceber sua participação na experiência que vive, enfrentar seus problemas de forma realística, visualizar alternativas de solução para seus problemas, testar novos padrões de comportamento, comunicar-se, socializar-se e aceitar encontrar um sentido em sua doença. A análise destes objetivos torna possivel concluir que a enfermagem constitui um processo de ajuda.

Entre os conceitos que MOSER (1970) considera básicos para as interações enfermeira-paciente está a mútua compreensão da linguagem entre terapêuta e paciente.

DAVITZ (1970) e ALSTSCHUL (1970) comentam a necessidade de encorajor enfermeiras a se comunicarem efetivamente com seus pacientes.

Autores como KREUTER (1957), BURSTEIN \& DIERS (1964), CONANT (1965), HAYS \& LARSON (1966 e 1970), LEWIS (1969), TRAVELBEE (1971), MANFREDA (1973) e PRUEN (1979), entre outros, afirmam ser a comunicação que ocorre na interação enfermeira-paciente fundamental, essencial e parte integrante do cuidado de enfermagem.

PATERSON \& ZDERARD (1976) colocam a enfermagem humanística como um diálogo, pois toda ação de enfermagem envolve uma forma especial de encontro de pessoas. ANDERSON (1979) também afirma que a enfermagem é um diálogo e destaca, ainda, a importância da comunicação não-verbal.

A comunicação eficaz, que é importante em todas as áreas da atividade humana, assume conotação mais ampla no planejamento do cuidado da saúde, pois a ansiedade experimentada pela pessoa doente torna-a mais vulnerável às mensagens verbais e não-verbais do terapeuta (DAUBENMIRE et alii, 1978).

Para HENDERSON \& NITE (1978) a habilidade no uso da comunicação por enfermeiras e outros profissionais da área de saúde é indispensável, tanto para percep̧ão dos aspectos emocionais como para desenvolvimento do relacionamento de ajuda com o paciente; esse relacionamento afeta todo procedimento de enfermagem e médico, contribuindo para o bem-estar do paciente. Os autores alertam os leitores, ainda, sobre a importância de todos os profissionais da área de saúde estarem conscientes de que, enquanto estão comunicando algo, quer verbal quer não verbalmente, eles estão automaticamente "ensinando" e os pacientes "aprendendo".

Em pesquisa realizada em uma unidade de terapia intensiva, NOBLE (1979) estudou os diferentes estímulos provenientes de aparelhos, luzes, tratamentos dolorosos, proximidade entre pacientes e a comunicação entre membros da equipe. Estes haviam sido citados como elementos que contribuíam para a chamada "síndrome ou psicose de terapia intensiva"; chegou à conclusão de que a comunicação dos membros da equipe, principalmente aquela que ocorre entre eles, é a principal responsável pelos distúrbios emocionais apresentados pelos pacientes.

Para NORDMARK \& ROHWEDER (1979), o equilibrio psicológico do individuo requer um meio adequado de comunicação com os outros que o cercam; 
consideram, ainda, que a eficácia do cuidado de enfermagem mantém relação direta com a habilidade de comunicação da enfermeira.

AGUILLERA (1977) e SMITH (1979) abordam a importância de ajudar o paciente a se valorizar como pessoa e a necessidade de oferecer-lhe oportunidades para se comunicar com outras pessoas que queiram ajudá-lo, a fim de que ele possa desenvolver relacionamento funcional dentro da sociedade.

EDWARDS \& BRILHART (1981) são de opinião que a habilidade interpessoal da enfermeira é tão importante para sua responsabilidade profissional quanto a habilidade técnica do cuidado direto. Ser capaz de comunicar-se adequadamente com o paciente pode ser uma questão de vida ou morte para esta pessoa.

\section{COMUNICAÇÃO TERAPÊUTICA}

Podemos inferir da revisão de literatura feita até aqui que a enfermeira tem a responsabilidade de tornar terapêutica sua comunicação com o paciente.

Comunicação terapêutica para RUESCH (1964) é aquela na qual o terapêuta tem por objetivo provocar mudança na forma de comunicação do outro o paciente - para produzir relações sociais mais gratificantes. $\mathrm{Na}$ comunicação com o paciente, o terapêuta ajuda-o a esclarecer aspectos conflitantes e oferece-lhe apoio, procurando torná-lo mais capaz de enfrentar seus problemas e de aceitar aquilo que não pode ser mudado. Para que a comunicação seja positiva ela tem que respeitar características como flexibilidade, propriedade, eficiência e resposta; a presença destas quatro características determina o sucesso da comunicação.

Interação positiva ou efetiva é facilitada quando há liberdade entre os interlocutores para perguntas e respostas, a fim de chegarem a melhor compreensão do que estão tentando comunicar; quando há respeito pelas idéias do outro; e, quando a comunicação não-verbal é percebida e interpretada acuradamente. Finalmente, comunicação é efetiva quando aumenta a compreensão entre os homens (LEWIS, 1969).

Entre outros autores, PEPLAU (1952), DAVIS (1963), SCHULLMAN et alii (1964), TRAVELBEE (1969), EDWARDS \& BRILHART (1981) acreditam que a habilidade em técnicas terapêuticas, na relação interpessoal, somente pode ser dominada com a prática e que só conhecimento teórico não é suficiente.

Consoante KRON (1972), a enfermeira, no desempenho de seu papel, usa continuamente alguma forma de comunicação. Deve ser considerada também a comunicação que é transmitida por gestos e expressão façial. É por meio desses que são comunicados, ao outro, os sentimentos, as emoçốes e as atitudes. A comunicação é a principal tarefa da enfermeira e é um processo contínuo. A chave para mantê-la deve ser usada, se se deseja compreender o outro e ser compreendido. A regra básica para comunicação efetiva ou terapêutica é usar a palavra certa, no momento certo, para a pessoa certa, no lugar certo e de mado certo. 
SIMONS (1969) ressalta a importância da enfermeira desenvolver meios de avaliar sua efetividade no uso da comunicação com pacientes.

Existe número reduzido de pesquisas sobre a qualidade do relacionamento enfermeira-paciente, como afirma PLUCHAN (1970).

De acordo com KRIZINOFSKY (1976) a aplicação da teoria da comunicação no campo da enfermagem geral, no da psiquiatria, com evidência na área de saúde mental, tem sido, historicamente, aspecto integrante do processo de enfermagem. As técnicas utilizadas na comunicação com paciente são chamadas de terapêuticas e têm sido preconizadas como base do relacionamento enfermeira-paciente. Algumas pesquisas têm evidenciado a diminuição da ansiedade pelo uso destas técnicas, mas, sobre a articulação da teoria de comunicação com o processo de enfermagem, muito pouco tem sido publicado.

Nesta breve revisão de literatura sobre a importância da comunicação na assistência de enfermagem, procuramos citar autores que pertencem a diversas áreas de enfermagem: enfermagem médica, cirúrgica, de educação e de ensino, de fundamentos de enfermagem e psiquiátrica; o objetivo foi evidenciar que a comunicação efetiva entre enfermeira e paciente é um dos meios mais valiosos de que esta profissional pode lançar mão para tornar o cuidado integral do paciente uma realidade, qualquer que seja sua área de atuação.

$E$ freqüente o paciente não apresentar resposta satisfatória mesmo que tratado com terapêutica adequada. Em geral, esta ocorrência é devida a problema emocional que repercute em seu organismo e impede sua pronta recuperação; a enfermeira, pelo uso adequado da comunicação, poderá identificar o problema real e ajudá-lo a recobrar a saúde.

Mesmo nas tarefas mais elementares de enfermagem, tais como oferecer comadre ao paciente, mudá-lo de decúbito, ajudá-lo a locomover-se, é por meio da comunicação com o paciente que a enfermeira transmite-lhe apoio, atenção, enfim trata-o como ser humano. Com isso ele terá satisfação de suas necessidades básicas de segurança, confiança e auto-estima, o que facilitará sua recuperação.

\section{CONSIDERAÇÕES FINAIS}

Quase todos os autores citados ressaltam a importância da comunicação não verbal e da ação desta sobre o paciente. De nada adianta dizermos ao paciente, por exemplo, como deve ser sua postura, se a nossa não está de acordo com o que comunicamos verbalmente. Da observação da interação enfermeira-paciente ou estudante-paciente podemos perceber que o paciente está, em geral, até mais atento à comunicação não verbal do que à verbal. Podemos extrapolar este princípio para o relacionamento professor-aluno. Este, como o paciente, está atento, o tempo todo, para a comunicação não verbal daquele, que deve ser coerente e consistente com a verbal.

Cremos ser indispensável que todas as escolas de enfermagem dêem ênfase a este aspecto da assistência de enfermagem. 0 ideal seria incluir, no currículo, uma disciplina no início do tronco profissonal comum, para atender a esta finalidade - relacionamento ou comunicação enfermeira-paciente. 
STEFANELLI, M. C. Importance of the communication process for nursing practice. Rev. Esc. Enf. USP, São Paulo, 15(3):239-245, 1981.

The Author presents a survey of the literature about the importance of communication in the nursing-patient interaction regardless to the area of practice. The author summarizes opinions about communication, therapeutic communication concepts and the need of nursing research in this area.

\section{REFERENCIAS BIBLTOGRAFICAS}

AGUILERA, D. C. Components of psychiatric nursing. In: - Review of psychiatric nursing. Saint Louis, Mosby, 1977. cap. 3, p. 37-58.

ALSTCHUL, A. T. Go and talk to the patients. Nursing Mirror, 130 (15): 41.6, 10 April 1970.

ANDERSON, N. D. Human interaction for nurses. Superv. Nurse, Chicago, 10 (10): 44, 48-50, Oct. 1979.

BURSTEN, B. \& DIERS, D. K. Pseudo-patient, centered orientation. Nurs. Forum, Hillsdale, 3 (2): $38-51,1964$.

CONANT, H. C. Use of Bales' interaction process analysis to study nurse-patient interaction. Nurs. Res., New York, 14 (4): 304-9, Fall 1965.

DAVIS, A. J. The skills of communication. Amer. J. Nurs., New York, 63 (1): 66-70, Jan. 1963.

DAVITZ, L. J. Introduction. In: - Interpersonal processes in nursing. Case histories. New York, Springer, 1970. cap. 1. p. 1-10.

FDWARDS, B. J. \& BRILHART, J. K. Nursing as communicating. In: - Communication in nurse practice. Saint Louis, C.V. Mosby, 1981. cap. 1. p. 1-23.

FUERST, E. V. \& WOLFF, L. Using communication skills. In: — Fundamentals of nursing. 3 ed. Philadelphia, J.B. Linpincott, 1964. part 17, p. 203-11.

GREGG, D. E. The psychiatric nurse's role. Amer. J. Nurs, New York, 54 (7): 848-51, July 1954.

GREGG, D. E. The therapeutic role of nurse. Perspect. Psychiatr. Care, Hillsdale, 1 (1): 18-24, Jan./ Feb. 1963.

HAYS, J. S. Analysis of nurse-patient communication. Nurs. Outlook, New York, 14 (9): $32-5,1966$.

HAYS, J. S. \& LARSON, K. Interacting with patients. New York, Macmillan, 1970. $282 \mathrm{p}$.

HENDERSON, V. \& NITE, G. Communication, human relations, learning, health goals and guidance. In: - Principles and practice of nursing. New York, Macmillan, 1978. cap. 16. p. 911-91.

HOFLING, C. $K$. et alii. Compreensión de las relaciones entre enfermera y paciente. In: Enfermeria psiquiátrica. 2.ed. México, Interamericana, 1970. cap. 3, p. 23-60.

KEMPF, F. C. Integrative behavior in the development of personality. In: - The person as nurse. New York, Macmillan, 1957. cap. 6. p. 92-115.

KREUTER, F. R. What is good nursing care. Nurs. Outlook, New York, 5 (5): 302-4, May 1957.

KRIZINOFSKY, M. T. Evolution of the communication model of therapy. In: 2 . Kneisl, C. R. \& WILSON, H. S. Current perspectives in psychiatric nursing. Saint Louis, Mosby, 1976. cap. 14, p. 148-63.

KRON, T. Communication in nursing. Philadelphia, W.B. Saunders, 1972. p. 1-12, 44-66, 284-290.

LEWIS, G. K. Communication. In: - Nurse-patient communication. 2.ed. Dubuque, Brown; 1969. p. 1.12, 58.72.

MANFREDA, L. M. Communication skills. In: —_. Psychiatric nursing. 9.ed. Philadelphia, Davis, 1973. cap. 21 , p. 262.76 .

MEYERS, M. E. The effects of types of communication on patients' reactions to stress. Nurs. Res., New York, $13(2)$ : 126-31, Spring 1964.

MORRIM, H. C. Communicating with and about patients and ther families. In: - Communication for nurses. Totowa, Littlefield, Adams, 1965. cap. 7. p. 76-90.

MOSER, D. H. Communicating with a schizophrenic patient. Perspect. Psychiatr. Care, Hillsdale, 8 (1): $36-45,1970$.

NIGHTINGALE, F. Notes on nursing. 2.ed. Philadelphia, Lippincott, 1946. p. 54-76.

NORDMARK, M. T. \& ROFWEDER, A. W. Comunicación. In: - Bases clentificas de la enfermería. 2.ed. México, Prensa Médica Mexicana, 1979. cap. 4, p. 585-93.

PEPLAU, H. E. Interpersonal relations in nursing. New York, Putnam's, 1952. p. 3-16, 263-309.

PLUCHAN, M. L. The nurse-patient relationship in the home setting. Ann Arbor, 1970. (Dissertation Faculty of the Graduate School of Arts and Sciences, University of Denver).

RUESCH, J. Disturbed communication. 5. ed. New York, W.W. Norton, 1957, p. 73

RUESCH, J. Comunicación terapéutica. Buenos Aires, Paidós, 1964 . 399 p.

SCHULMAN, $M$. D. et alii. The therapeutic dialogue: a method for the analysis of verbal interaction. Springfield, Charles C. Thomas, 1964. p. 8-16, 87-115.

SIMMONS, J. A. Observation and collection of data. In: —_. The nurse-patient relationship in psychiatric nursing. Philadelphia, W.B. Saunders, 1969. cap. 2, p. 11-36.

SMITH, C. Communication skills. Nurs. Times, London, 75 (22): 926-9, 31 May 1979.

SULLIVAN, H. S. Definitions. In: The interpersonal theory of psychiatry. New York, W.W. Norton, 1953. cap. 2, p. 13-30.

TRAVELBEE, J. Intervention in psychiatric nursing: process in the one-to-one relationship. Philadelphia, F.A., Davis, 1969. $280 \mathrm{p}$.

phia, Davis, 1971. cap. 9. p. 93-116.

TUDOR, G. A sociopsychiatric nursing approach to intervention in a problem of mutual withdrawal on a mental hospital ward. Perspect. Psychistr. Care, Hillsdale, 8 (11): 11-35, Jan./Feb. 1970.

WIEDENBACH, E. The helping art of nursing. Amer. J. Nurs., New York, 63 (11): 54-7, Nov. 1963. 